\title{
Estimation of the threat of IEMI to complex electronic systems
}

\author{
R. Kanyou Nana ${ }^{1}$, S. Korte ${ }^{2}$, S. Dickmann ${ }^{1}$, H. Garbe ${ }^{2}$, and F. Sabath ${ }^{3}$ \\ ${ }^{1}$ Helmut-Schmidt-Universität/Universität der Bundeswehr Hamburg, Germany \\ ${ }^{2}$ Leibniz-Universität Hannover, Germany \\ ${ }^{3}$ Wehrwissenschaftliches Institut für Schutztechnologien, Munster, Germany
}

\begin{abstract}
The threat of ultra wideband (UWB) sources is interesting for military issues. This paper summarizes information concerning the voltages generated from some commercially available UWB generator systems and their produced electromagnetic fields. The paper focuses on the coupling of UWB fields into electronic equipment and discusses possible modeling and measurement techniques to estimate such a threat for modern ships. An evaluation procedure for the determination of the induced voltage at the input of an electronic component is presented. This method is based on the computation of the internal electric field and the measurements on a test network, which is similar to the structure of the steering control cabling. It allows the estimation of the potential threat for the ship's electronic equipment due to the exposal to UWB emitting sources.
\end{abstract}

\section{Introduction}

In complex systems like ships or aircrafts many tasks vital to the function of the system are executed by electronic equipment. Earlier research from Ianoz and Wipf (2000) as well as Radasky (2006) has shown, that there are frequency ranges in many of these systems, where disturbances in the system will be observed if an external electromagnetic field exceeds a certain limit. This induced effect in an electronic equipment is commonly known as IEMI (intentional electromagnetic interference). In order to understand the threats to modern ships, it is necessary to define the electromagnetic environment, that can cause operational problems for exposed ship systems. With respect to criminal purposes e.g. terrorism, it is useful to consider an intentional electromagnetic environment (IEME) created by UWB generator systems. For

Correspondence to: R. Kanyou Nana (kanyounana@hsu-hh.de) this type of threat, a produced pulse typically has frequency components over a wide spectral range. Due to the fact that many systems have resonances that create significant susceptibilities to particular frequencies as mentioned above, it is possible that ship systems are affected by the voltages and currents caused by incident UWB fields. This can cause disturbances or failures in these systems which could lead to severe problems.

The structure of this paper is as follows: the modeling of the problem is presented in Sect. 2. Starting with the voltages from some commercially available UWB generator systems, a brief overview concerning the derivation of the incident fields is given in Sect. 3. After that, in Sect. 4, modeling and measurement techniques for the description of the different coupling mechanisms into the considered system, here the chassis of the steering control and an Ethernet test network, are discussed. Aspects for the estimation of UWB threats for marine equipment, like the computation of the internal fields and the induced disturbance voltage at the input of an electronic component, are in the focus of Sect. 5.

\section{Modeling of the problem}

The problem faced here is illustrated in Fig. 1. It shows a frigate irradiated by a transient electromagnetic field of an

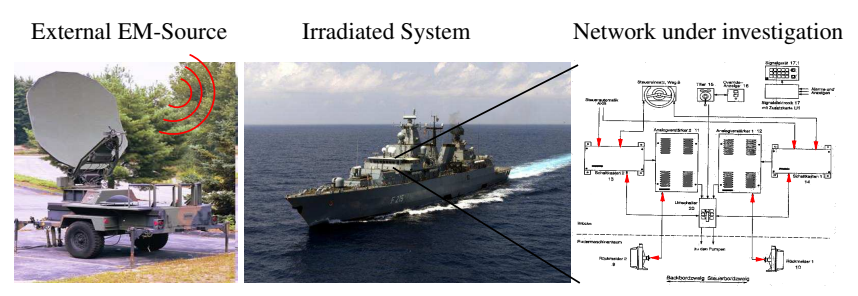

Fig. 1. Geometry of the system, which is irradiated by a transient electromagnetic field of an external interfering source. 


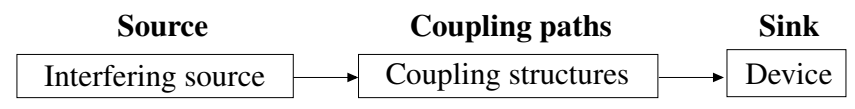

Fig. 2. Coupling interference as a typical EMC problem.

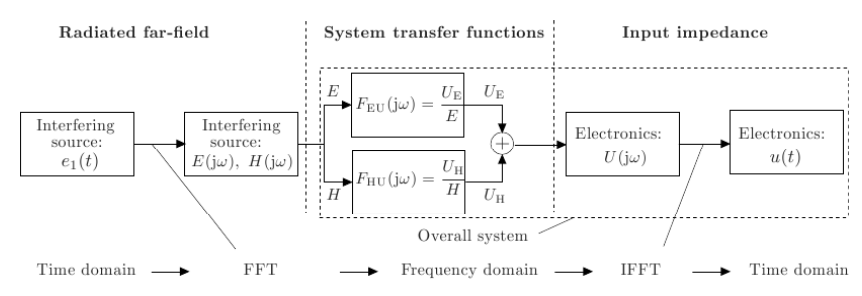

Fig. 3. Detailed description of the coupling interference.

external UWB interfering source. The determination of the coupling into an internal network of this system is a typical EMC problem which can be devided into three parts: source, coupling paths and sink as shown in Fig. 2.

The determination of the radiated electric far-field $E_{1}(\mathrm{j} \omega)$ is suitable for the characterisation of the external interfering source. Therewith the needed incident electromagnetic field can be calculated:

$$
E(\mathrm{j} \omega)=E_{1}(\mathrm{j} \omega) \frac{r_{1}}{r} ; \quad H(\mathrm{j} \omega)=\frac{E(\mathrm{j} \omega)}{Z_{0}}=\frac{r_{1} \cdot E_{1}(\mathrm{j} \omega)}{r \cdot Z_{0}},
$$

where $Z_{0}$ represents the free space wave impedance. $E_{1}(\mathrm{j} \omega)$ stands for the (known) radiated electric far-field evaluated at a distance $r_{1}$ from the interfering source and $(E(\mathrm{j} \omega), H(\mathrm{j} \omega))$ are the incident electromagnetic field, which must be determined at the distance $r$ from the interfering source (placed directly in front of the investigated system, here the ship). The coupling paths are generally modelled with system transfer functions. Since most devices connected to the examined network have metallic enclosures, it can be assumed that electromagnetic disturbances predominantly couple into the electronic devices through the transmission lines. Therefore the input impedance of the device adequately describes it as the sink. A detailed partitioning of the initial problem is shown in Fig. 3.

This approach is based on the knowledge of the radiated electric far-field in time domain $e_{1}(t)$, which makes it possible to determine the spectra of the incident electromagnetic field $E(\mathrm{j} \omega), H(\mathrm{j} \omega)$ by using the FFT (Fast Fourier Transform) and the formulas in (1). Because the electric as well as the magnetic part of the field contribute to the overall coupling in the definition domain of the system transfer functions, the coupling of the external electromagnetic field into the electronics can be described in the frequency domain by separated system transfer functions $F_{\mathrm{EU}}(\mathrm{j} \omega)$ and $F_{\mathrm{HU}}(\mathrm{j} \omega)$. They establish the interrelationship between the external electromagnetic field $E(\mathrm{j} \omega), H(\mathrm{j} \omega)$ and the fraction of voltages at the terminals of the electronic components $U_{\mathrm{E}}(\mathrm{j} \omega), U_{\mathrm{H}}(\mathrm{j} \omega)$ in the investigated network. The product

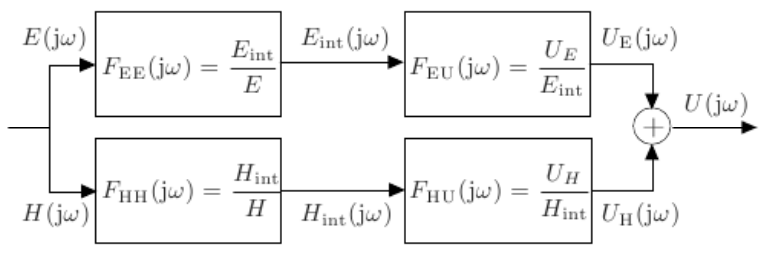

Fig. 4. Decomposition of the system transfer functions illustrated in Fig. 3 in field $\left(F_{\mathrm{EE}}(\mathrm{j} \omega), F_{\mathrm{HH}}(\mathrm{j} \omega)\right)$ and coupling transfer functions $\left(F_{\mathrm{EU}}(\mathrm{j} \omega), F_{\mathrm{HU}}(\mathrm{j} \omega)\right)$.

of both fractions of the field $E(\mathrm{j} \omega)$ and $H(\mathrm{j} \omega)$ with the corresponding system transfer function $F_{\mathrm{EU}}(\mathrm{j} \omega)$ and $F_{\mathrm{HU}}(\mathrm{j} \omega)$ yields the fractional system responses $U_{\mathrm{E}}(\mathrm{j} \omega)$ and $U_{\mathrm{H}}(\mathrm{j} \omega)$. The superposition of these intermediate results leads to the system response in the frequency domain $U(\mathrm{j} \omega)$, which next can be used to obtain the system response in the time domain $u(t)$ by using the IFFT (Inverse Fast Fourier Transform).

Since $F_{\mathrm{EU}}(\mathrm{j} \omega)$ and $F_{\mathrm{HU}}(\mathrm{j} \omega)$ strongly depend on the geometry of the system, a structure modification in the system (i.e. the dimensions of the apertures or the load impedances) requires a complete new computation. It has been shown in Kanyou Nana (2008), that $F_{\mathrm{EU}}(\mathrm{j} \omega)$ rather $F_{\mathrm{HU}}(\mathrm{j} \omega)$ are made up of contributions from several coupling paths of electromagnetic fields, which are represented by different (elementary) transfer functions. In the scope of this problem the coupling through apertures predominates. Thus, for the determination of the coupled disturbance voltages, first the internal electromagnetic field $E_{\text {int }}(\mathrm{j} \omega), H_{\text {int }}(\mathrm{j} \omega)$ in a close vicinity of the network should be evaluated. This field is then used to excite the network (model based on the equivalent generators along the cables and the transmission lines of the network). Consequently the initial system transfer functions (Fig. 3) can be decomposed in field and coupling transfer functions (Fig. 4), which implies that a structure modification in the system solely requires a re-evaluation of the affected transfer functions.

The disadvantage of this method is that not only two but at least four transfer functions are necessary to perform a complete analysis of the problem. For network problems in which the electromagnetic field is considered as primary source, it has been proven in Tesche, Ianov and Karlsson (1996) that the effect of the magnetic field can be expressed by the electric field. This typical solution form of network problems is known as Agrawal method. For the present case, this means that only the knowledge of the internal electric field $E_{\text {int }}(\mathrm{j} \omega)$ is required for the computation of the disturbance voltage $U(\mathrm{j} \omega)$. Thus, the number of transfer functions can be reduced to the half as presented in Fig. 5. 


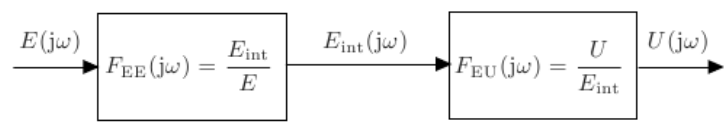

Fig. 5. Simplification of the transfer functions shown in Fig. 4 by making use of the Agrawal method.

Table 1. Analytical description of the voltages from some commercial UWB pulse generator systems.

\begin{tabular}{|c|c|c|c|c|}
\hline Pulse form & \multicolumn{3}{|c|}{ Time domain representation } & $u_{i \max }$ \\
\hline Half sine & $u_{1}(t)=\{$ & $=\left\{\begin{array}{l}u_{1 \max } \cdot \sin \left(\omega_{0} t\right) \\
0\end{array}\right.$ & $\begin{array}{l}\quad \frac{2 \pi}{\omega_{0}} \leq t \leq \frac{3 \pi}{\omega_{0}} \\
\text { sonst }\end{array}$ & $50 \mathrm{kV}$ \\
\hline Unipolar double exponential & $u_{2}(t)=\{$ & \multicolumn{2}{|c|}{$\begin{array}{ll}u_{2 \max } \cdot k(\alpha, \beta) \cdot\left(\mathrm{e}^{-\alpha t}-\mathrm{e}^{-\beta t}\right) & , \quad t \geq 0 \\
0 & , \quad t<0\end{array}$} & $45 \mathrm{kV}$ \\
\hline Full sine & $u_{3}(t)$ & $\begin{array}{l}u_{3 \max } \cdot \sin \left(\omega_{0} t\right) \\
0\end{array}$ & $\begin{array}{l}\frac{2 \pi}{\omega_{0}} \leq t \leq \frac{4 \pi}{\omega_{0}} \\
\text { sonst }\end{array}$ & $50 \mathrm{kV}$ \\
\hline
\end{tabular}

\section{Characterisation of the interfering source}

Voltages from commercial UWB pulse generators can be generally approximated with the analytical functions shown in Table 1.

Next it will be proven that the knowledge of the generator voltage enables a description of the radiated electric field and after that the derivation of an analytical expression for the electric far-field. Here the signal forms shown in Fig. 6 are considered, which correspond to the graphical representations of the pulses in Table 1: the half sine pulse, the unipolar double exponential pulse as well as the full sine pulse. The radiation of these signals can be realised with the following types of antennas:

- the TEM Horn antenna (Half and Full sine pulses), and

- the reflector antenna Type IRA (unipolar double exponential pulse).

Because antennas generally have a derivative behaviour, the radiated electric far-field is proportional to the time derivative of the generator voltage:

$$
e_{i}(t) \sim \frac{\mathrm{d} u_{i}(t)}{\mathrm{d} t} .
$$

The normalization of $\frac{\mathrm{d} u_{i}(t)}{\mathrm{d} t}$ with its maximum value consequently results in the normalized electric far-field $e_{i}(t) / e_{i \max }$. For the above-mentioned generator voltages, Fig. 7 illustrates the resulting electric field. A use of the FFT on $e_{i}(t) / e_{i \max }$ yields the normalized spectrum $E_{i}(\mathrm{j} \omega) / e_{i \max }$, the magnitude of which is shown in Fig. 8. The needed spectrum $E_{i}(\mathrm{j} \omega)$ results from the multiplication of the normalized spectrum with the maximum field value $e_{i \max }$ that depends on the type of antenna.
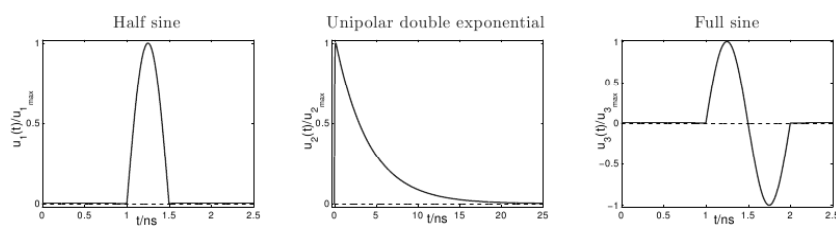

Fig. 6. Voltages from some commercial UWB generator systems.
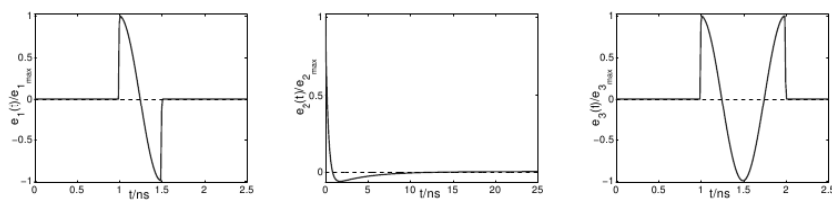

Fig. 7. Estimated normalized electric far-fields generated from some commercial UWB generator systems.

\section{Characterisation of the coupling structures}

For the characterisation of the coupling effects of electromagnetic field pulses into complex systems, the field transfer function $F_{\mathrm{EE}}(\mathrm{j} \omega)$ as well as the coupling transfer function $F_{\mathrm{EU}}(\mathrm{j} \omega)$ were introduced. A field transfer function is a function that transforms a field from one position (i.e. the external incident field) into another field on another position (i.e. the field inside the chassis of the steering control next to the network). A coupling transfer function is a function that converts a field from one position (i.e. the field inside the chassis of the steering control next to the network) into a voltage on a precise point of the network (i.e. the induced voltage at the device's input). In the following, these transfer functions will be quantified in order to describe the existing coupling structures of the investigated system.

\subsection{Modeling of the ship}

To compute the field transfer function $F_{\mathrm{EE}}(\mathrm{j} \omega)$, numerical simulations were performed using the 3D-FDTD based code PAM-CEM/FD. Due to the size and the complexity of the ship, we used a simplified model, in which only the bridge (which contents the chassis of the steering control) was taken into account, because it can be regarded as effectively isolated from the rest of the ship. The bridge is modeled as a metal cavity containing just the relevant metallic objects (Fig. 9). One of these objects is the steering control unit.

While modeling the chassis of the steering control, its basic structure and the internal big metallic objects have been taken into account. Smaller structures like complicated harnesses were been neglected. Since the coupling field in the chassis is generated through the slots, it was necessary to discretize the smallest dimension of each implemented slot with at least two patches. The used PAM-CEM/FD model of the chassis is depicted in Fig. 10. 

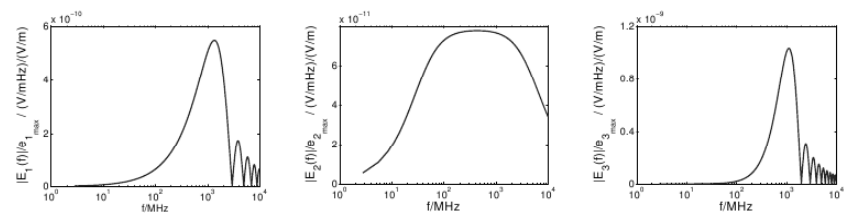

Fig. 8. Magnitude of normalized spectra of the estimated electric far-fields.

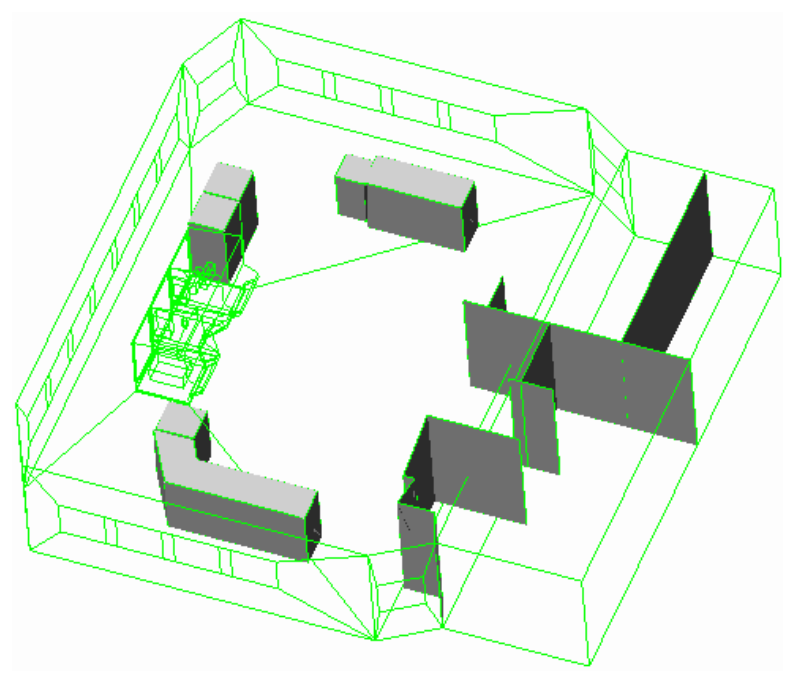

Fig. 9. A view of the simplified bridge model.

After modeling the different parts of the structure, it was necessary to define the type of the excitation of the system. The whole system was illuminated by a vertical and horizontal polarised electromagnetic field, respectively. Then the normalisation of the computed electric field inside the bridge with respect to the incident field yields to the simulated field transfer function.

Measurements were performed to validate the numerical modeling. A simplified block diagram of the measurement points and the instrumentation is shown in Fig. 11. The measurement for the evaluation $F_{\mathrm{EE}}(\mathrm{j} \omega)$ was performed in two steps. First a reference measurement was done without the ship and second the measurement was carried out on the ship, where all measuring instruments were placed inside the bridge except the transmitting antenna. The ratio between both measurement results yields to the desired transfer function. For point $\mathrm{P}_{1}$ the magnitude of $F_{\mathrm{EE}}(\mathrm{j} \omega)$ in the simulations and measurements, in the case of a vertical polarized field, are compared in Fig. 12. From a EMC point of view, both results agree quite well. Thus, the presented model can be used to estimate the field in the chassis of the steering control.

\subsection{Ethernet test network}

The coupling transfer function $F_{\mathrm{EU}}(\mathrm{j} \omega)$ was determined with measurements of an ethernet cable (type: cat 5) with an

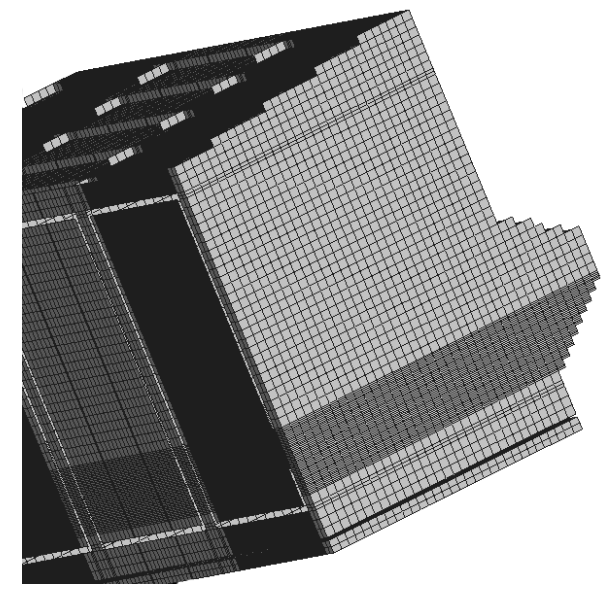

Fig. 10. PAM-CEM/FD model of the chassis of the steering control.
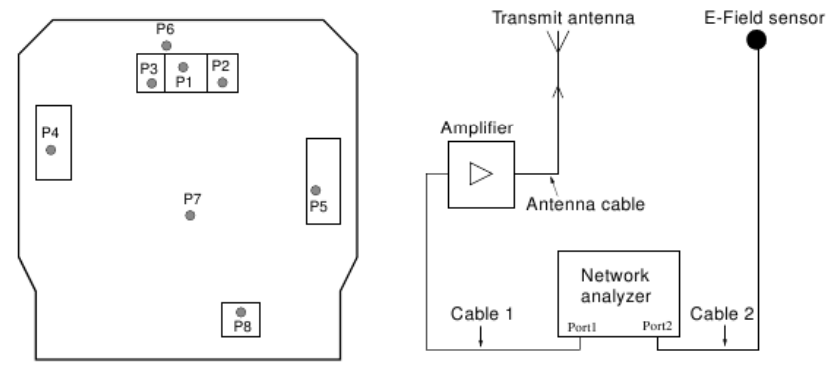

Fig. 11. Block diagram of measurement points and instrumentation for validation of the simplified bridge model.

overall length of $5 \mathrm{~m}$ and terminated with $100 \Omega$ load resistance. Its magnitude for different coupling lengths is presented in Fig. 13. As can be seen, the magnitude of $F_{\mathrm{EU}}(\mathrm{j} \omega)$ depends strongly on the coupling length and has frequency components only from $17 \mathrm{MHz}$ to $200 \mathrm{MHz}$. This frequency range contains the first resonance frequency of the used cable $(20 \mathrm{MHz})$, which can be calculated using the following formula $\left(\varepsilon_{r}=2.25, \mu_{r}=1\right)$ :

$$
f=\frac{c}{2 l \sqrt{\varepsilon_{r} \mu_{r}}} .
$$

\section{Estimation of the induced voltages}

With respect to the above-mentioned UWB antenna types, the peak value of the electric field, which can be achieved at a distance of about $1 \mathrm{~km}$ without exaggeration, are in the order of $50 \mathrm{~V} / \mathrm{m}$ for a TEM-horn $\left(30 \mathrm{~cm} \times 30 \mathrm{~cm} \times 30 \mathrm{~cm}, u_{\max }=50 \mathrm{kV}\right)$ and $34 \mathrm{~V} / \mathrm{m}$ for an IRA $\left(\varnothing: 90 \mathrm{~cm}, \mathrm{u}_{\max }=9 \mathrm{kV}\right)$. Such levels of fields should therefore be considered in order to assess the induced voltage on a $100 \Omega$ load resistance at the end of the ethernet cable. Multiplying these maximum field values with the corresponding normalized electric field from Fig. 8, the incident 


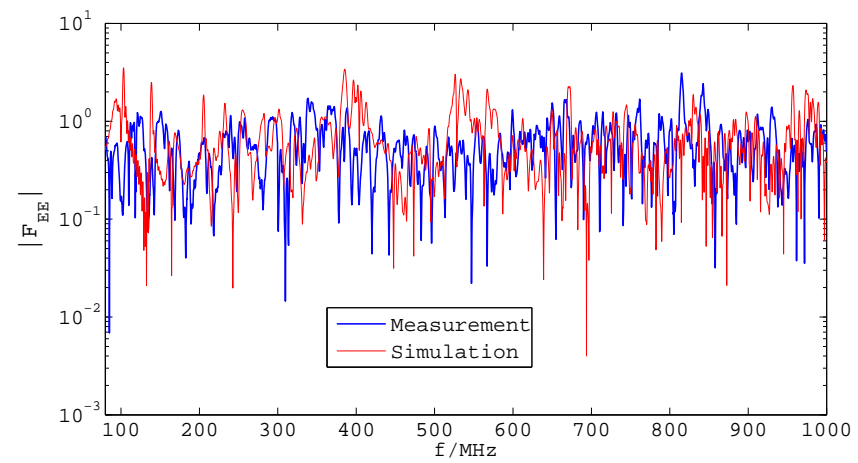

Fig. 12. Comparison of calculated and measured field transfer function at the point $\mathrm{P}_{1}$ inside the chassis of the steering control.

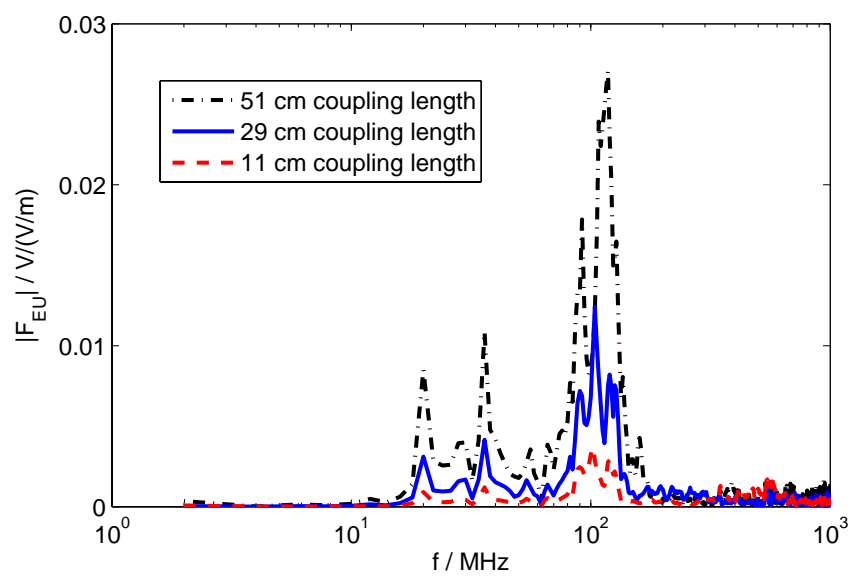

Fig. 13. Measured coupling transfer function of a $100 \Omega$ terminated ethernet cable for $51 \mathrm{~cm}, 29 \mathrm{~cm}$ and $11 \mathrm{~cm}$ coupling lengths.

electric field in the frequency domain can be estimated. A multiplication of this field with both the field transfer function and the coupling transfer function, and followed by IFFT, leads to the desired induced voltage. The results are presented in Fig. 14. As can be seen, induced voltages up to 0.06-0.1 V appear at the entry of a sensitive electronic equipment, which can lead to functional disturbances for some electronics.

\section{Conclusions}

With regard to commercially available UWB generator systems, a methodology for the derivation of the incident electric fields has been presented. With the help of these fields and by using both the field and the coupling transfer functions of the investigated system, a first estimation of the induced disturbance voltage at the entry of ship's electronics has been done. These results can be used to estimate the threat of such a system under IEMI.
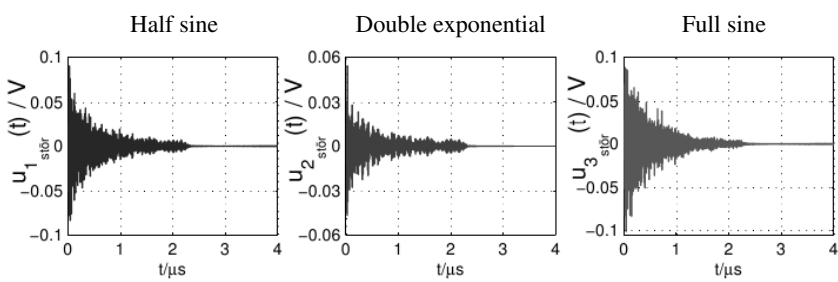

Fig. 14. Estimated induced voltage on a $100 \Omega$ load resistance for an $5 \mathrm{~m}$ length ethernet cable (coupling length: $51 \mathrm{~cm}$ ).

Acknowledgement. This work has been financed by the Bundesamt für Wehrtechnik und Beschaffung. The authors would like to thank P. Dietz of the Einsatzflottille 2 (German Navy) for his support.

\section{References}

Ianoz, M. and Wipf, H.: Modeling and Simulation Methods to Assess EM Terrorism Effects, Asia-Pacific Conference on Environmental Electromagnetics CEEM, Shanghai, China, 2000.

Radasky, W. A.: The Threat of Intentional Electromagnetic Interference (IEMI) to Wired and Wireless Systems, 17th International Zurich Symposium on Electromagnetic Compatibility, Zurich, Switzerland, 110-163, 2006.

Kanyou Nana, R., Dickmann, S., and Sabath, F.: Electromagnetic field vulnerability of complex systems - an application of EM topology, Adv. Radio Sci., 6, 273-277, 2008, http://www.adv-radio-sci.net/6/273/2008/.

Tesche, F. M., Ianov, M. V., and Karlsson T.: EMC Analysis Methods and Computational Models, ISBN: 0-471-15573-X, John Wiley \& Sons, Inc., New York, USA, 1996.

Camp, M.: Empfindlichkeit elektronischer Schaltungen gegen transiente elektromagnetische Feldimpulse, ISBN 3-8322-3504-3, Shaker Verlag, Dissertation, Universität Hannover, 2004.

Sonnemann, F.: Susceptibility Investigations of High-Power EMFields on electronic systems, International Symposium and technical Exhibition on electromagnetic Compatibility, Zurich, Switzerland, 2003.

Baum, C. E.: Electromagnetic Topology: A formal approach to the analysis and design of complex electronic systems, Proc. Zurich EMC Symp., 209-214, 1982.

Paul, C. R.: Analysis of Multiconductor Transmission Lines, ISBN 0-471-02080-X, John Wiley \& Sons, Includes bibliographical references and index, New York, USA, 1994.

Giri, D. V.: Radiation of impulse-like waveforms with illustrative applications, Ultrawideband and Ultrashort Impulse Signals, Sevastopol, Ukraine, 19-22, September 2004.

IEEE Std. 299: IEEE Standard Method for Measuring the Effectiveness of Electromagnetic Shielding Enclosures, IEEE Inc., 345 East 47 Street, New York, NY 10017, USA, 1997. 